\title{
Angle Tracking Observer for Filtering Rotor Position Estimates in Sensorless Electric Drives
}

\author{
Leszek Jarzebowicz ${ }^{1}$, Maciej Cisek $^{1}$, Artur Opalinski ${ }^{1}$ \\ ${ }^{I}$ Faculty of Electrical and Control Engineering, Gdansk University of Technology, \\ G. Narutowicza 11/12,80-233 Gdansk, Poland \\ leszek.jarzebowicz@pg.gda.pl
}

\begin{abstract}
Sensorless electric drives use measurements of electrical quantities to estimate rotor position and speed. Measurement errors deteriorate the estimation accuracy, thus additional means are necessary to remove high-frequency components from estimated signals. This paper proposes to use an Angle Tracking Observer (ATO) for reducing noise in rotor position estimates, which is related to random errors in the measurements. A simplified, universal structure of ATO has been designed for this purpose. Based on simulations, the gains of ATO algorithm have been selected for optimal balance between error components related to the measurement noise and to the filtering lag. Additionally, implementation issues have been discussed and laboratory validation has been carried out in the paper. The designed ATO is applicable to any sensorless drive and enables drive controller to substantially improve rotor estimation accuracy using very low computing overhead.
\end{abstract}

Index Terms-Filtering algorithms; motion estimation; noise cancellation; observers; sensorless control; variable speed drives.

\section{INTRODUCTION}

Sensorless control of electric drives, which replaces position and speed sensor with an estimation algorithm, is intensively researched [1], [2]. Most sensorless drives estimate position and speed using model-based approach. Numerous observers have been proposed, which complement motor model with various methods of correcting its state variables according to measured voltages and currents [3]. In observers no additional means are required to filter the estimates, because the balance between good dynamic properties of the state variables and the sensitivity to measurement noise can be controlled by adjusting gains in the feedback matrix.

In contrast to the model-based sensorless approach, there are also estimation algorithms which identify selected motor parameters, e.g. stator inductances, whose values are directly related to rotor position [4]. As the estimates are based solely on the values identified in the last control cycle, any measurement noise is immediately reflected onto estimated position waveform. Consequently, low-pass filtering is typically used before applying estimated position to drive control algorithm [5], [6]. A lag (phase shift) in the position signal, related to filtering, remains negligible as long as these estimators are used at low speeds. However,

Manuscript received 21 March, 2016; accepted 14 July, 2016. selected parameter-based estimators are dedicated to medium and high rotor speeds. In such case, specific means of noise reduction are required to condition the signal of estimated position. High-speed operation is very susceptible to control lags, thus standard low-pass filtering is not appropriate for this task.

This paper proposes applying an Angle Tracking Observer (ATO) to improve estimation accuracy of the parameter-based estimator presented in [7]. ATOs have been widely researched in regard to conditioning signals from position transducers. However, ATO structures from the literature are designed for distinctive input signals and thus cannot be directly applied to rotor position estimator.

The digest of current ATO solutions is presented in Section II. A proposed structure of new ATO, dedicated for filtering rotor position estimates, is described in Section III. A mathematical model of electric drive is presented in Section IV, and later used for pre-selecting ATO gains and evaluating filtering effectiveness, as described in Sections V and VI. Finally, implementation issues and experimental results are included in Section VII, and conclusions are presented in the last section.

\section{REVIEW OF Present ANGLE Tracking OBSERVERS}

ATOs are widely applied in integrated circuits used for determining angular position from mechanical transducers called resolvers. The circuits are referred to as Resolver-toDigital (RDC) converters.

ATO reduces impact of disturbances in analog signals, provided by resolver, on the estimated angular position [8], [9]. ATO achieves this without introducing the lag related to angular speed. The functional diagram of a typical hardware-based ATO is shown in Fig. 1.

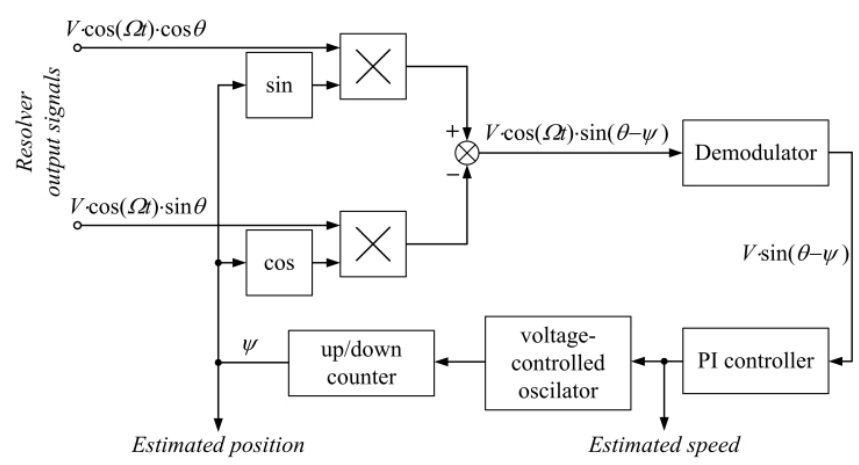

Fig. 1. Typical structure of Angle Tracking Observer [9] 
Signals provided by a resolver are related to cosine and sine of measured angular position $\theta$. These signals are modulated by carrier signal $V \times \cos (\Omega t)$ from a reference generator. ATO multiplies the modulated signals by sine and cosine, respectively, of the observed angular position $\psi$. Subtracting the resulting signals, followed by removing the carrier $\cos (\Omega t)$, produces error signal $V \times \sin (\theta-\psi)$ for a PI controller. As $\theta-\psi$ difference is assumed small, the error signal may be approximated as $V \times(\theta-\psi)$. This linear input signal enables the PI controller to provide output related to angular speed, which is next transformed to a variable frequency signal that drives an up/down counter. Since the counter state representing the estimated angular position $\psi$ is updated in single step increments, thus the estimate changes smoothly.

The typical ATO structure has been subjected to numerous improvements. Hoseinnezhad et al. proposed a hybrid observer structure consisting of ATO and quadrature encoder [8]. This solution improves stability of the observer at high speed and at high acceleration. Benammar and Gonzales modified the ATO tracking loop by applying a window comparator and an amplifier with controlled phase inversion [9]. The main advantage of this improvement is a reduction of manufacturing costs of integrated circuits. Sivappagari and Konduru introduced an ATO that applies synchronous demodulation of the input signals and rectangular reference signal [10]. Moreover, the authors proposed quadrature estimation of initial rotor position to improve the precision of position determination.

Software implementation of ATO algorithms has been investigated in [11], [12]. These solutions allow for extraction of the angular position without dedicated hardware circuits, decreasing production costs. Zhao, Qiao and $\mathrm{Wu}$ proposed to apply ATO for improving rotor position estimation accuracy in a sensorless drive [13]. As their estimator delivers $\sin \theta$ and $\cos \theta$ signals, it is well suited for the input of a typical ATO structure.

\section{Proposed Structure of NeW Angle Tracking OBSERVER}

In sensorless electric drives it is essential to limit peak error of estimated position used by drive control algorithm. The filtering procedure should be computationally simple not to lose the primary advantage of parameter-based estimators, which is very short execution time.

The review of the research on ATO shows that currently there is no solution that can be directly applied to a typical parameter-based rotor position estimator. To make the ATO directly applicable to the above, the following issues have to be considered:

1. Adapting the observer to a position-related signal, instead of currently used sine and cosine signals;

2. Enabling software implementation without complex operations, like trigonometric function calculation, division or multiplication.

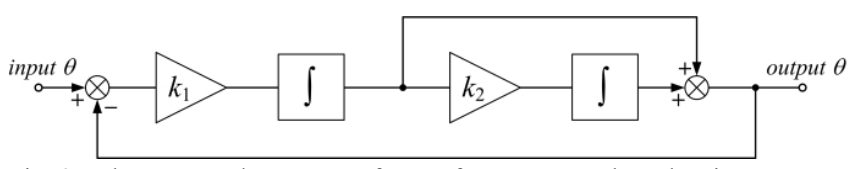

Fig. 2. The proposed structure of ATO for parameter-based estimators.
The proposed ATO is shown in Fig. 2. A second-order structure has been chosen to supress the speed-dependent errors. The counter has been replaced with an integrator. The input stage has been simplified and adapted to the position-related signal.

\section{Simulation MODEL}

Properties of the proposed ATO are investigated using a MATLAB/Simulink model. The general structure of the model is presented in Fig. 3. The model consists of motor mechanical dynamics subsystem which computes rotor position and rotor speed from the waveform of motor electromagnetic torque, by using the following equations:

$$
\begin{gathered}
\frac{d \omega_{m}}{d t}=\frac{1}{J}\left(T_{e}-T_{r}\right), \\
\frac{d \theta_{m}}{d t}=\omega_{m},
\end{gathered}
$$

where $\omega_{m}$ - rotor speed, $\theta_{m}-$ rotor position, $J$ - rotor moment of inertia, $T_{e}$ - motor electromagnetic torque, $T_{r}-$ resistance torque related to friction. All the symbols correspond to mechanical (not electrical) quantities.

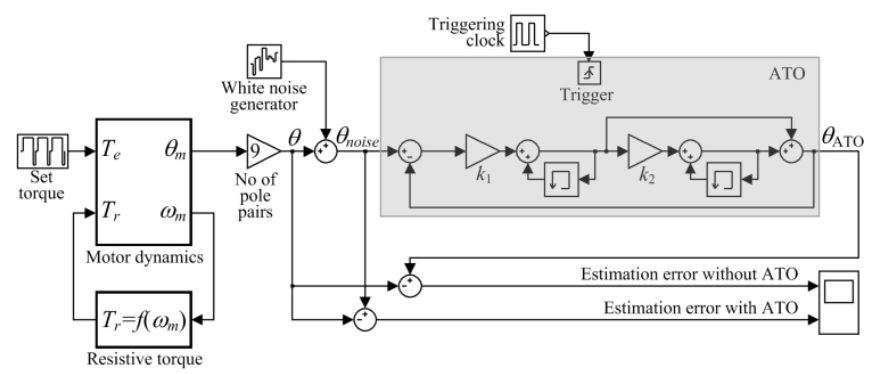

Fig. 3. Simulation model for investigating and verifying ATO.

The parameters of the mechanical dynamics subsystem correspond to a laboratory drive used later for experimental validation (Table I). The resistance torque was derived empirically, as a function of rotor speed, and approximated by a quadratic function. The moment of inertia was determined experimentally [14] for the set of three mechanically-coupled electric motors used in validation.

TABLE I. SELECTED PARAMETERS OF THE LABORATORY DRIVE.

\begin{tabular}{|c|c|}
\hline Parameter & Value \\
\hline Rated power $P_{n}$ & $1.1 \mathrm{~kW}$ \\
\hline Number of pole pairs $p$ & 9 \\
\hline Base speed (electrical) $\omega_{b}$ & $1000 \mathrm{rad} / \mathrm{s}$ \\
\hline Maximal speed (electrical) $\omega_{m}$ & $1200 \mathrm{rad} / \mathrm{s}$ \\
\hline Rated torque $T_{n}$ & $10.4 \mathrm{Nm}$ \\
\hline Moment of inertia $J$ & $0.19 \mathrm{~kg} \cdot \mathrm{m}^{2}$ \\
\hline Control algorithm execution period & $100 \mu \mathrm{s}$ \\
\hline
\end{tabular}

To reproduce the noise in the estimated position signal, caused by the measurement errors, the modelled electrical rotor position $\theta=p \times \theta_{m}$ is supplemented with white noise. The modelled laboratory drive consists of rotor position estimator from [7], which uses motor phase current derivatives to compute estimates. Using current derivatives results in simple computations, but the $d i / d t$ measurements are noisy [15], [16], exerting significant impact on the estimates. Thus the power of the white noise applied in the 
model is relatively high in order to represent the properties of the laboratory drive used later for validation. The noised position signal $\theta_{\text {noise }}$ is delivered to the ATO's input.

The structure of the ATO corresponds to the one from Fig. 2; however, it reflects digital implementation. The integration is performed with rectangular approximation and the execution of the ATO is triggered by $10 \mathrm{kHz}$ clock, similarly to the drive controller in the modelled laboratory stand [17].

\section{Methodology of Pre-Selecting ATO Gains}

The effectiveness of noise filtering is ultimately dependent on ATO gains $k_{1}$ and $k_{2}$. High gains cause poor noise suppression and may result in loosing stability of the observer. In contrast, small gains result in a smooth output signal, but also introduce distinctive speed and accelerationrelated signal errors on the output.

On the basis of the simulation model, a procedure to preselect the gains for the laboratory drive is proposed. As it is assumed that drive control stability is related to instantaneous position errors, the aim of tuning is to find ATO gains that minimize peak estimation errors defined as the difference between ATO output signal $\theta_{A T O}$ and the real rotor angular position $\theta$.

Since the errors consist of components related to speed, acceleration and jerk, the analysis has to consider maximal values of these parameters as well as their different compositions. A dedicated motor torque cycle, as shown in Fig. 4(a), is proposed to meet these requirements. It repeats interleaving periods of propelling and braking with rated torque. Since duration of this cycle is dominated by propelling intervals, the speed gradually increases. The waveform of the estimation error corresponding to the torque cycle, obtained for optimal ATO gains, is shown in Fig. 4(b).

The results of analysing the impact of gains $k_{1}$ and $k_{2}$ on peak estimation errors are presented in Fig. 5. The lowest peak error of $0.8 \%$ was obtained for $k_{1}=k_{2}=2.3 \times 10^{-6}$; however, there is a relatively wide range of gains corresponding to errors less than $1 \%$ (marked with dark grey colour).

\section{COMPARISON OF ATO AND LOW-PASS FILTERS}

Two common FIR filters are used to contrast performance of the proposed solution: a moving average (boxcar) filter and a sinc filter with Blackman window. Selected FIR filtering methods are linear-phase, but the obvious method of preserving phase by using negative indexes of filter coefficients is not applicable when processing signals in real-time. Thus, to restore the phase, the position signal is assumed linear in the filtering window and extrapolated to compensate the phase lag.

Boxcar and sinc filters, with and without phase compensation, are used to de-noise the position signal $\theta_{\text {noise }}$ from the simulation model. Their window length $w$ and sinc cut-off frequency $f_{c}$ were tuned in order to minimize the peak error in the position signal, which is the main quality indicator in the considered application, as pointed out in Section III.

A comparison of RMS and peak errors of considered filtering methods is presented in Fig. 6. The uncompensated FIR filters do not provide a significant noise reduction, because their window lengths must be kept small in order to limit the phase delay (lag). The effectiveness of their phasecompensated variants is close to the one of ATO; however, it comes with a significant processing load, as convolution with larger filter kernel $(w>100)$ must be performed in each control cycle.
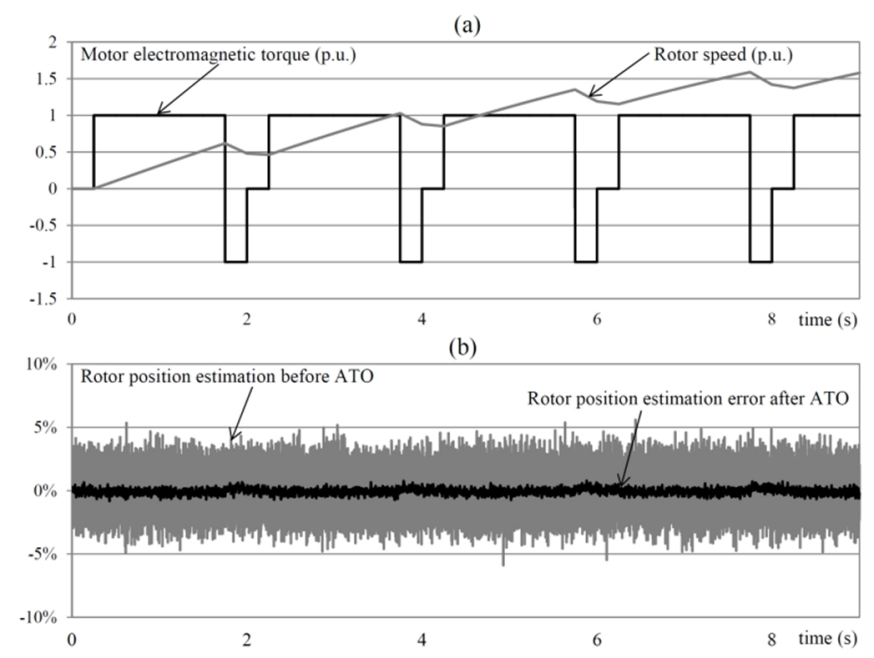

Fig. 4. Simulation results obtained for $k_{1}=k_{2}=2.3 \cdot 10^{-6}$ : motor electromagnetic torque and speed (a), rotor position estimation obtained with and without applying the ATO (b).

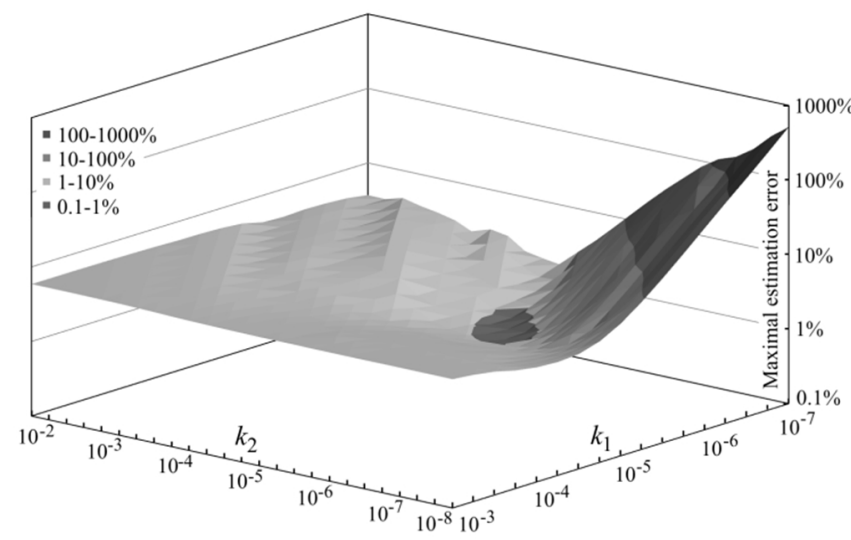

Fig. 5. Relation between peak rotor position estimation error and ATO gains $k_{1}, k_{2}$ for the proposed drive operating cycle.
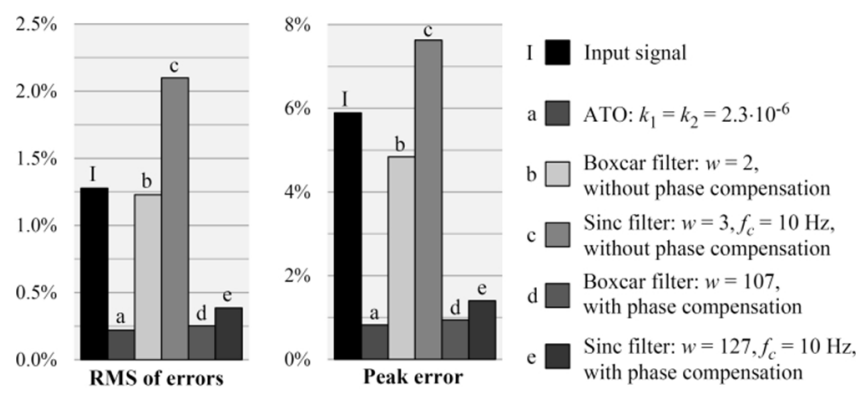

Fig. 6. Comparison of estimation errors for different filtering algorithms.

\section{IMPLEMENTATION AND EXPERIMENTAL VALIDATION}

The effectiveness of applying the ATO is investigated using the parameter-based estimator from [7] and the laboratory drive whose parameters are given in Table I. The drive is equipped with a resolver and an integrated Resolverto-Digital converter to measure the accurate rotor position, which is necessary for determining estimation errors. The 
ATO was included into the sensorless control algorithm code of drive controller based on TMS320F2812 processor.

Experimental verification uses the same torque cycle as simulation (Fig. 4(a)), although the real torque generated by the laboratory drive is limited when operating above the base speed due to power limitation (see Fig. 7(a)). ATO gains were fine-tuned under the criterion of minimum peak error for the test-cycle based operation. The optimal gains for the laboratory drive $k_{1}=k_{2}=0.8 \times 10^{-6}$ slightly differ from the pre-selected ones: $k_{1}=k_{2}=2.3 \times 10^{-6}$, but they lie within the optimal area identified in simulation (Fig. 5). The estimation errors for the fine-tuned gains are presented in Fig. 7(b).

(a)

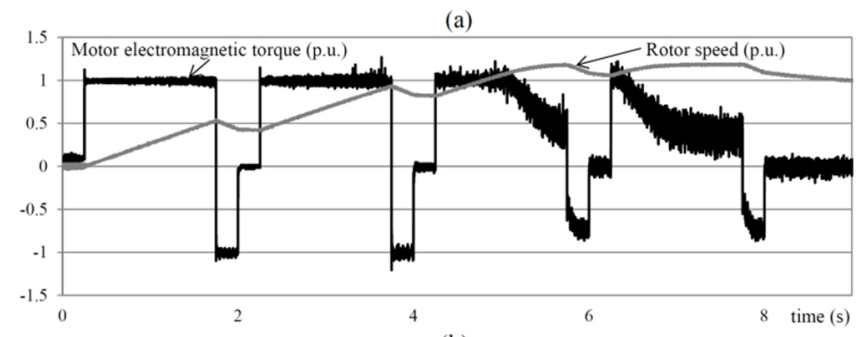

(b)

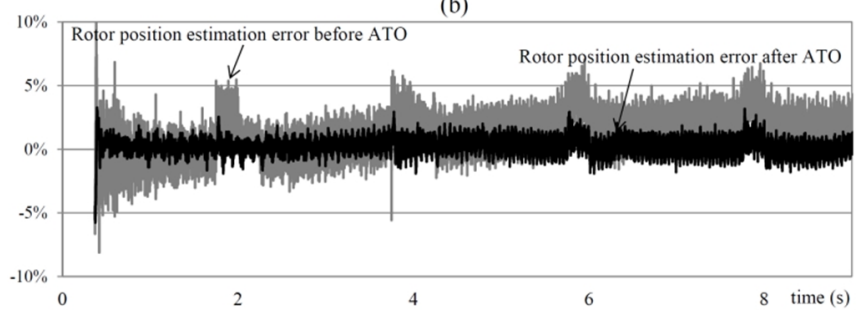

Fig. 7. Experimental results obtained for $k_{1}=k_{2}=0.8 \cdot 10^{-6}$ : motor electromagnetic torque and speed (a), rotor position estimation error obtained with and without applying the ATO (b).

Selecting the gains which fulfil $k=C \times 2^{n}$, where $C$ is control cycle duration and $n$ is an integer, allows for replacing multiplication operation by bit shifting, which accelerates the computations. The ATO is implemented using the following code:

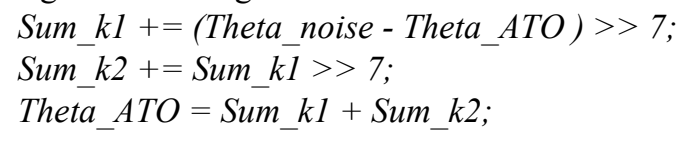

\section{CONCLUSIONS}

The paper contributes: a new ATO structure; a methodology of pre-selecting observer gains based on simulation model; a comparison of performance of the proposed solution with common digital low-pass filters; a software implementation of the ATO and its experimental validation.

The ATO is proposed to fulfil specific requirements of sensorless drives for filtering rotor position estimates. The model and the gain selection procedure allow for obtaining initial gains, to be fine-tuned in a target system. Software implementation of the proposed ATO structure is straightforward and uses fast instructions. As the ATO is adapted to position-related signal, in contrast to referred solutions based on sine and cosine signal, it may be applied to a wide range of sensorless drives.

\section{REFERENCES}

[1] J. Guzinski, H. Abu-Rub, "Speed sensorless induction motor drive with predictive current controller", IEEE Trans. Industrial Electronics, vol. 60, no. 2, pp. 699-709, 2013. [Online]. Available: http://dx.doi.org/ 10.1109/TIE.2012.2205359

[2] Y. S. Kung, N. P. Thanh, M. S. Wang, "Design and simulation of a sensorless permanent magnet synchronous motor drive with microprocessor-based PI controller and dedicated hardware EKF estimator", Applied Mathematical Modelling, vol. 39, no. 19, pp. 5816-5827, 2015. [Online]. Available: http://dx.doi.org/ 10.1016/j.apm.2015.02.034

[3] A. Zaafouri, F. Farhani, A. Chaari, "Robust observer design with pole placement constraints for induction motor control", Elektronika ir Elektrotechnika, vol. 21, no. 1, pp. 18-22, 2015. [Online]. Available: http://dx.doi.org/10.5755/j01.eee.21.1.5223

[4] M. Seilmeier, B. Piepenbreier, "Sensorless control of PMSM for the whole speed range using two-degree-of-freedom current control and HF test current injection for low-speed range", IEEE Trans. Power Electronics, vol. 30, no. 8, pp. 4394-4403, 2015. [Online]. Available: http://dx.doi.org/ 10.1109/TPEL.2014.2353215

[5] N. R. M. Juremi, M. A. Zulkifley, A. Hussain, "Second order polynomial filter as filtering technique in steady state visual evoked potential's control signal", in Proc. Electronics, Information and Communications (ICEIC), 2014. [Online]. Available: http://dx.doi.org/10.1109/ELINFOCOM.2014.6914381

[6] Z. Shunyi, J. Pomarico-Franquiz, Y. S. Shmaliy, "An approach to nonlinear state estimation using extended FIR filtering", in Proc. Signal Processing Conference (EUSIPCO), 2014, pp. 436-440.

[7] L. Jarzebowicz, "Sensorless IPMSM drive with rotor position estimator based on analysis of phase current derivatives", in Proc. Int. Symposium on Industrial Electronics (ISIE), 2011, pp. 733-738. [Online]. Available: http://dx.doi.org/10.1109/ISIE.2011.5984248

[8] R. Hoseinnezhad, P. Harding, "A novel hybrid angle tracking observer for resolver to digital conversion", in Proc. $44^{\text {th }}$ IEEE Conf. Decision and Control (CDC-ECC), 2005, pp. 7020-7025. [Online]. Available: http://dx.doi.org/10.1109/CDC.2005.1583292

[9] M. Benammar, A. S. Gonzales, "A novel resolver converter based on a modified tracking method", in Proc. 10th IEEE Int. Conf. Networking, Sensing and Control (ICNSC), 2013, pp. 586-590 [Online]. Available: http://dx.doi.org/10.1109/ICNSC.2013.6548804

[10] Sivappagari C. M. R., Konduru N. R., "Modified ATO algorithm based high accuracy RDC using pulse excitation", in Proc. First International Conference Automation, Control, Energy and Systems (ACES), 2014, pp. 1-5. [Online]. Available: http://dx.doi.org/ 10.1109/ACES.2014.6808034

[11] D. A. Khaburi, "Software-based resolver-to-digital converter for DSPbased drives using an improved angle-tracking observer", IEEE Trans. Instrumentation and Measurement, vol. 61, no. 4, pp. 922-929, 2012. [Online]. Available: http://dx.doi.org/10.1109/TIM.2011. 2179825

[12] L. Idkhajine, E. Monmasson, M. W. Naouar, A. Prata, "Fully integrated FPGA-based controller for synchronous motor drive", IEEE Trans. Industrial Electronics, vol. 56, no. 10, pp. 4006-4017, 2009. [Online]. Available: http://dx.doi.org/10.1109/TIE.2009. 2021591

[13] Y. Zhao, W. Qiao, L. Wu, "Position extraction from a discrete sliding-mode observer for sensorless control of IPMSMs", in Proc. 2012 IEEE Int. Symposium on Industrial Electronics (ISIE), 2012, pp. 725-730. [Online]. Available: http://dx.doi.org/10.1109/ISIE. 2012.6237159

[14] T. Fedor, J. Vittek, P. Sindler, "Influence of variable moment of inertia in robot servo motor control", in Proc. ELEKTRO, 2014, pp. 165-169. [Online]. Available: http://dx.doi.org/ 10.1109/ELEKTRO.2014.6847894

[15] R. Czerwinski, T. Rudnicki, "Examination of electromagnetic noises and practical operations of a PMSM motor driven by a DSP and controlled by means of field oriented control", Elektronika ir Elektrotechnika, vol. 20, no. 5, pp. 46-50, 2014. [Online]. Available: http://dx.doi.org/10.5755/j01.eee.20.5.7099

[16] R. Dolecek, O. Cerny, J. Novak, M. Bartlomiejczyk, "Interference in power system for traction drive with PMSM", Przeglad Elektrotechniczny - Electrical Review, no. 9a, pp. 204-207, 2012.

[17] L. Jarzebowicz, "Indirect measurement of motor current derivatives in PMSM sensorless drives", Elektronika ir Elektrotechnika, vol. 20, no. 7, pp. 23-26, 2014. [Online]. Available: http://dx.doi.org/ 10.5755/j01.eee.20.7.8019 